\title{
ESTUDO DAS RELAÇÕES ENTRE AS TAXAS DE GLICOSE NO LIQÜIDO CEFALORRAQUEANO E NO SANGUE
}

\author{
Horacio M. Canelas * \\ A. Spina frança Netto * \\ Josí Axtonio Levy* \\ Maria Irmina Valente* \\ J. M. T. Bittexcourt*
}

Apesar da importância da glicorraquia dentro da semiologia liquórica, não dispomos ainda de conhecimento exato sôbre a influência que as injeções intravenosas de glicose hipertônica - de uso corrente no tratamento da hipertensão intracraniana - teriam sôbre o teor de glicose do líqüido cefalorraqueano. Freqủentes vêzes fica-se na dúvida se a verificação de hiperglicorraquia em pacientes submetidos a essa terapêutica terá significado patológico, ou se ela constituirá mera conseqüência da introdução de glicose no organismo. Em outras eventualidades, fatos inversos se observam: ignora-se, por exemplo, o preciso valor diagnóstico que se poderá emprestar à taxa de glicose no líquor de pacientes suspeitos de neurotuberculose submetidos àquele tratamento.

No sentido de procurar esclarecer esta questão, resolvemos realizar o presente trabalho. Aproveitamos o material de que dispúnhamos para abordar os problemas referentes às relações entre as taxas de glicose no sangue e no líquor, e também para estudar a possível influência dos processos meningo-parenquimatosos sôbre a permeabilidade da barreira hemoliquórica ao glicídeo.

Estes problemas já vêm sendo investigados há muito. O valor do quociente entre as taxas de glicose no líquor e no sangue varia grandemente, em condições normais, segundo vários autores, podendo-se considerar como índices médios limítrofes os valores 1,13 (Polonovski e Duhot ${ }^{1}$ ) e 0,37 (Wilcox e col. ${ }^{2}$ ). Nizna e outros ${ }^{3}$ denominaram "coeficiente de permea-

Trabalho da Clínica Neurológica da Fac. Med. da Univ. de São Paulo (Serviço do Prof. Adherbal Tolosa) e do Laboratório Central do Hospital das Clínicas de São Paulo (Chefe: Dr. O. A. Germek).

* Assistentes.

Nota dos autores - Agradecemos ao Dr. Lindo Fava, assistente do Departamento de Estafística da Faculdade de Filosofia, Ciências e Letras da Univ. de São Paulo, que nos orientou na realização da análise estatística; e às técnicas da Secção de Liquiido Cefalorraqueano do Laboratório Central do Hospital das Clínicas de São Paulo, Izette Caldeira Cardoso da Cunha e Teresinha Rocha. Freire, pela dedicada colaboração que nos prestaram. 
bilidade" à relação inversa, cujo valor médio seria de 1,60 segundo Tcherkassov e Jolkver ${ }^{4}$. Este coeficiente, para Nizna, seria normalmente invariável nos casos de hiper ou hipoglicemia, mas diminuiria nos casos de meningite; contudo, Tcherkassov e Jolkver observaram, surpreendentemente, que, em tais condições, êle aumentava. 0 trabalho dêstes autores foi realizado da seguinte forma: era provocada uma hiperglicemıi, sendo então colhidos, simultâneamente, sangue e líquor; não cogitaram de estudar as curvas glicêmica e da glicorraquia.

Por outro lado, Becker ${ }^{5}$ estudou, em neuroluéticos, o efeito da ingestão de $100 \mathrm{~g}$ de dextrose, fazendo colheitas simultâneas de sangue e líquor 15,30 e 60 minutos após; verificou que, enquanto a glicorraquia não se modificava, a glicemia atingia um máximo 30 minutos após a ingestão. Mas Dietel ${ }^{6}$, utilizando a administração oral e intravenosa de açúcar, pôde comprovar que o aumento da taxa de glicose no líquor sempre se seguia, após certo intervalo, à elevação da glicemia. Schjött ${ }^{7}$ observou que o aumento da taxa de glicose no líquor se iniciava de 90 a 120 minutos após a ingestão de açúcar, atingindo o máximo depois de 150 a 180 minutos; em epilépticos êsse aumento era mais precoce e mais intenso. Merritt e Fremont-Smith ${ }^{8}$ fizeram a administração intravenosa de glicose em 3 rasos, observando, 60 a 90 minutos após, aumento de 10 a $33 \%$ em relação à taxa inicial do hidrocarbonado.

$\mathrm{Na}$ revisão da literatura não encontramos, porém, trabalhos nos quais tivesse sido verificada sistemàticamente a relação entre as curvas glicêmica e da glicorraquia; geralmente, era colhida apenas uma amostra de líquor de cada indivíduo em estudo, após a determinação da taxa inicial. Graças ao método por nós empregado, cremos que a questão foi investigada de maneira original, com maior amplitude e com mais precisão, dado o tratamento estatístico a que foram submetidos nossos resultados.

\section{MATERIAL}

Nosso material é constituído por três grupos de pacientes: Grupo 1 - Três pacientes que serviram de contrôle e nos quais não foi injetada glicose, sendo, porém, colhidas amostras de sangue e líquor de acôrdo com a norma geral. Grupo $\mathscr{2}$ - Dez pacientes em que foram administradas injeções de glicose em dose comparável às habitualmente usadas em clínica, isto é, de $10 \mathrm{~g}$ (20 $\mathrm{ml}$ de soluto a 50\%). Esta série foi dividida em dois subgrupos: A, 5 pacientes com líquor normal (casos 4 a 8) e B, 5 pacientes com líquor alterado (casos 9 a 13). Grupo 3 - Vinte pacientes em que foram injetadas, na veia, $50 \mathrm{~g}$ de glicose (100 $\mathrm{ml}$ de soluto a 50\%). Esta série também foi dividida em dois subgrupos: A, 10 pacientes com líquor normal (casos 14 a 23) e B, 10 pacientes com líquor alterado (casos 24 a 33). Consideramos como alterados os líquores que apresentavam, isolada ou associadamente, hipercitose, hiperproteinorraquia, alterações de reações coloidais e específicas. Em nenhum dos casos havia manifestações que indicassem a existência de distúrbios no metabolismo dos hidratos de carbono. Evidentemente, a existência de hipertensão intracraniana ou de bloqueio do canal raqueano foi considerada como contra-indicação para a feitura da prova. 


\section{METODOS}

Em todos os pacientes procedemos, inicialmente, à colheita concomitante, em jejum, de sangue e líquor. Imediatamente após, era feita a injeção intraveriosa de glicose. Novás amostras simultâneas de sangue e líquor eram colhidas após $15,30,45,60,120,180$ e 240 minutos. Durante todo êsse tempo a agulha de punção era conservada em posição, não sendo repetida a raquicentese com a finalidade de evitar uma acidental "contaminação" do líquor com sangue. No dia seguinte, em jejum, em 10 casos do grupo 3, foi realizada nova retirada concomitante de líquor e sangue. Para a injeção de glicose e colheita de sangue foi utilizada, geralmente, uma veia da região da prega do cotovêlo; o líquor foi sempre colhido por punção lombar, estando o paciente em decúbito lateral. Com fins profiláticos, administrou-se antibióticos após a prova.

As determinações da glicemia e da glicorraquia foram efetuadas pelo método de Folin e $\mathrm{Wu} 9$, sendo o material prèviamente desproteinizado com ácido sulfúrico $\mathrm{N} / 12$ e tungstato de sódio a $10 \%$; leitura colorimétrica em espectrofotômetro de Coleman, com comprimento de onda de $0,420 \mu$. Resultados em mg/ $100 \mathrm{ml}$.

Êrro do método - No sentido de determiná-lo, lançamos mão do seguinte processo. De um mesmo indivíduo (DDO, reg. HC 219769) foi colhida, em cois dias diferentes, uma amostra de aproximadamente $40 \mathrm{ml}$ de líquor. Êsse ìotal foi dividido em 8 tubos e enviado ao Laboratório Central, sem qualquer referência especial, como se se tratasse de uma prova igual às outras e cada tubo contivesse amostras retiradas na ordem cronológica referida. Obtivemos, para a $1^{\text {a }}$ collheita, os seguintes resultados: $75,77,75,75,75,70,70,70$. Para a $2^{\text {a }}$ colheita, as dosagens foram: 72, 84, 86, 81, 84, 79, 75, 75. Portanto, como valores médios: $73,5 \quad 80,5 \quad 80,5 \quad 78,0 \quad 79,5 \quad 74,5 \quad 72,5 \quad 72,5$. Os coeficientes de variação relativos às duas colheitas foram elevados $(3,925$ e 6,403). Entretanto, a prova de homogeneidade (Snedecor 10a), isto é, a relação entre as variâncias de ambas as colheitas, demonstrou um valor de $F$ não significante ao nível fiducial de $2 \%$. Efetuamos o mesmo teste entre os valores médios do paciente DDO e os dos grupos 1, 2 e 3, obtendo, respectivamente, os seguintes resultados: 4,163 1,992 19,583. A penas êste último foi significante (valor de $F$ ao nível fiducial de $2 \%$ para $n_{1}-n_{2}=7$, igual a 7,000). Quer isto dizer que as oscilações das dosagens verificadas no caso DDO (êrro do método), no grupo 1 e no grupo 2 são comparáveis; e que as referentes ao conjunto de 20 casos do grupo 3 foram nitidamente diversas das atribuíveis simplesmente ao êrro experimental.

Evidenciado o elevado êrro do método, resolvemos verificar se, nos trís grupos, as taxas de glicose no líquor sof riam variações interrelacionadas diretamente com o momento da colheita. Nesse sentido, após comprovar a homogeneidade inicial das amostras pela análise de variância, calculamos e comparamos as equações de regressão linear das taxas de glicose em relação ao tempo, nos vários grupos. Estudamos também, nos grupos 2 e 3, o comportamento dos quociertes entre a taxa de glicose no líquor e no sangue (L/S) no decurso das provas. Estudamos o valor inicial dêsse quociente, em nosso material, fazendo a comparação entre os grupos com líquor normal e os com líquor alterado. Finalmente, confrontamos os valores iniciais da glicemia e da glicorraquia, com os obtidos após $2,3,4$ e 24, horas, no grupo 3 .

\section{RESULTADOS}

Evolução da glicorraquia no grupo 1 - Nos 3 casos dêste grupo não foi administrada glicose, sendo, entretanto, colhidas amostras concomitan- 


\begin{tabular}{|c|c|c|c|c|c|c|c|c|c|c|}
\hline \multirow{2}{*}{$\begin{array}{c}\text { CASO } \\
\text { REG.BC }\end{array}$} & \multirow[t]{2}{*}{ HUNORES } & \multicolumn{9}{|c|}{ AMOSTRAS (tejpo ex minutos) } \\
\hline & & 0 & 15 & 30 & $\leq \equiv$ & fo & 320 & 180 & 240 & $24 \mathrm{hs}$. \\
\hline $\begin{array}{l}1(J G 0) \\
255833\end{array}$ & $\begin{array}{l}\text { Liquor } \\
\text { Sangue }\end{array}$ & $\begin{array}{l}53 \\
85\end{array}$ & $\begin{array}{l}49 \\
75\end{array}$ & $\begin{array}{l}59 \\
85\end{array}$ & $\begin{array}{l}54 \\
75\end{array}$ & $\begin{array}{l}54 \\
77\end{array}$ & $=-$ & $\begin{array}{l}40 \\
80\end{array}$ & $\begin{array}{l}53 \\
80\end{array}$ & $=$ \\
\hline $\begin{array}{l}2(\mathrm{JAF}) \\
256961\end{array}$ & $\begin{array}{l}\text { Liquor } \\
\text { Sangue }\end{array}$ & $\begin{array}{l}54 \\
87\end{array}$ & $\begin{array}{l}61 \\
85\end{array}$ & $\begin{array}{l}63 \\
83\end{array}$ & $\begin{array}{l}50 \\
85\end{array}$ & $\begin{array}{l}59 \\
75\end{array}$ & $\begin{array}{l}50 \\
70\end{array}$ & $\begin{array}{l}53 \\
80\end{array}$ & $\begin{array}{l}50 \\
88\end{array}$ & $=$ \\
\hline $\begin{array}{l}3 \text { (JRF) } \\
256161\end{array}$ & $\begin{array}{l}\text { Liquor } \\
\text { Sangue }\end{array}$ & $\begin{array}{l}53 \\
83\end{array}$ & $\begin{array}{l}42 \\
80\end{array}$ & $\begin{array}{l}42 \\
80\end{array}$ & $\begin{array}{l}40 \\
75\end{array}$ & $\begin{array}{l}43 \\
83\end{array}$ & $\begin{array}{l}48 \\
77\end{array}$ & $\begin{array}{l}48 \\
79\end{array}$ & $\begin{array}{l}39 \\
80\end{array}$ & -- \\
\hline MEDINS & $\begin{array}{l}\text { ifquor } \\
\text { Sargue }\end{array}$ & $\begin{array}{l}53,3 \\
85,0\end{array}$ & $\begin{array}{l}50,7 \\
80,0\end{array}$ & $\begin{array}{l}54,7 \\
82,7\end{array}$ & $\begin{array}{l}48,0 \\
78,0\end{array}$ & $\begin{array}{l}52,0 \\
78,3\end{array}$ & $\begin{array}{l}49,0 \\
73,5\end{array}$ & $\begin{array}{l}50,0 \\
79.7\end{array}$ & $\begin{array}{l}47,3 \\
82,7\end{array}$ & -- \\
\hline
\end{tabular}

Quadro 1 - Resultado das dosagens de glicose no líquor e no sangue, nos pacientes do grupo 1 (nos quais não foi injetada glicose).

\begin{tabular}{|c|c|c|c|c|c|c|c|c|c|c|}
\hline \multirow{2}{*}{$\begin{array}{c}\text { CASO } \\
\text { REG.HC }\end{array}$} & \multirow[t]{2}{*}{ EUNORES } & \multicolumn{9}{|c|}{ AMOSTRAE (temio om mimutos) } \\
\hline & & 0 & 15 & 30 & 45 & 60 & 120 & 180 & 240 & $24 \mathrm{hg}$. \\
\hline $\begin{array}{l}4 \text { (K) j } \\
220169\end{array}$ & $\begin{array}{l}\text { Elquor } \\
\text { Sangue }\end{array}$ & $\begin{array}{l}69 \\
94\end{array}$ & $\begin{array}{r}40 \\
112\end{array}$ & $\begin{array}{r}44 \\
106\end{array}$ & $\begin{array}{l}50 \\
85\end{array}$ & $\begin{array}{l}46 \\
--\end{array}$ & $\begin{array}{l}45 \\
--\end{array}$ & $\begin{array}{l}44 \\
83\end{array}$ & $\begin{array}{r}34 \\
106\end{array}$ & 58 \\
\hline $\begin{array}{l}5 \text { (JI) } \\
330344\end{array}$ & $\begin{array}{l}\text { Lfquor } \\
\text { Sangoe }\end{array}$ & $\begin{array}{r}45 \\
104\end{array}$ & $\begin{array}{r}45 \\
163\end{array}$ & $\begin{array}{r}50 \\
129\end{array}$ & $\begin{array}{r}50 \\
103\end{array}$ & $\begin{array}{r}57 \\
i 21\end{array}$ & $\begin{array}{l}49 \\
--\end{array}$ & $\begin{array}{l}63 \\
91\end{array}$ & $\begin{array}{l}48 \\
97\end{array}$ & $m$ \\
\hline $\begin{array}{l}6 \quad(J P S) \\
332423\end{array}$ & $\begin{array}{l}\text { Llquor } \\
\text { Sangue }\end{array}$ & $\begin{array}{r}60 \\
126\end{array}$ & $\begin{array}{r}6 \varepsilon \\
142\end{array}$ & $\begin{array}{r}61 \\
100\end{array}$ & $\begin{array}{r}57 \\
101\end{array}$ & $\begin{array}{r}60 \\
129\end{array}$ & $\begin{array}{l}46 \\
--\end{array}$ & $\begin{array}{r}49 \\
111\end{array}$ & $\begin{array}{r}45 \\
117\end{array}$ & - \\
\hline $\begin{array}{l}7 \text { (BA) } \\
169681\end{array}$ & $\begin{array}{l}\text { Lfquor } \\
\text { Sangue }\end{array}$ & $\begin{array}{l}50 \\
64\end{array}$ & $\begin{array}{r}51 \\
212\end{array}$ & $\begin{array}{l}51 \\
72\end{array}$ & $\begin{array}{l}53 \\
8 i\end{array}$ & $\begin{array}{l}51 \\
80\end{array}$ & $\begin{array}{l}44 \\
83\end{array}$ & $\begin{array}{l}46 \\
83\end{array}$ & $\begin{array}{l}42 \\
77\end{array}$ & - \\
\hline $\begin{array}{l}8(J V C) \\
332698\end{array}$ & $\begin{array}{l}\text { ifquor } \\
\text { Sarmue }\end{array}$ & $\begin{array}{l}76 \\
-\end{array}$ & $\begin{array}{l}125 \\
141\end{array}$ & $\begin{array}{l}70 \\
81\end{array}$ & $\begin{array}{l}69 \\
88\end{array}$ & $\begin{array}{r}69 \\
106\end{array}$ & $\begin{array}{l}73 \\
90\end{array}$ & $\begin{array}{r}69 \\
112\end{array}$ & $\begin{array}{r}69 \\
111\end{array}$ & -- \\
\hline $\begin{array}{l}\text { MEDIAS } \\
\text { HORMAIS }\end{array}$ & $\begin{array}{l}\text { Liquor } \\
\text { Sangue }\end{array}$ & $\begin{array}{l}58,0 \\
\$ 7,0\end{array}$ & $\begin{array}{r}65.8 \\
134,0\end{array}$ & $\begin{array}{l}55, \hat{z} \\
97,6\end{array}$ & $\begin{array}{l}57,8 \\
91,6\end{array}$ & $\begin{array}{r}56,0 \\
111,5\end{array}$ & $\begin{array}{l}51,6 \\
86,5\end{array}$ & $\begin{array}{l}54,2 \\
95,0\end{array}$ & $\begin{array}{r}47,6 \\
101,6\end{array}$ & -- \\
\hline $\begin{array}{l}9(\mathrm{AB}) \\
147687\end{array}$ & $\begin{array}{l}\text { Lfquor } \\
\text { Sangue }\end{array}$ & $\begin{array}{r}57 \\
141\end{array}$ & $\begin{array}{r}54 \\
157\end{array}$ & $\begin{array}{l}54 \\
--\end{array}$ & $\begin{array}{r}59 \\
129\end{array}$ & $\begin{array}{l}65 \\
97\end{array}$ & $\begin{array}{l}6 ! \\
97\end{array}$ & $\begin{array}{l}53 \\
97\end{array}$ & $\begin{array}{l}50 \\
97\end{array}$ & $\begin{array}{l}50 \\
94\end{array}$ \\
\hline $10(A C)$ & Liquer & 53 & 57 & 50 & $\begin{array}{l}57 \\
75\end{array}$ & $\begin{array}{l}53 \\
77\end{array}$ & $\begin{array}{l}54 \\
85\end{array}$ & $\begin{array}{l}54 \\
85\end{array}$ & $\begin{array}{l}43 \\
88\end{array}$ & 63 \\
\hline $\begin{array}{l}11 \quad(\mathrm{Rb}) \\
228439\end{array}$ & $\begin{array}{l}\text { Llyuor } \\
\text { Sartue }\end{array}$ & $\begin{array}{l}63 \\
83\end{array}$ & $\begin{array}{r}61 \\
122\end{array}$ & $\begin{array}{r}59 \\
100\end{array}$ & $\begin{array}{l}59 \\
94\end{array}$ & $\begin{array}{l}59 \\
91\end{array}$ & $\begin{array}{l}59 \\
97\end{array}$ & $\begin{array}{r}59 \\
103\end{array}$ & $\begin{array}{r}59 \\
106\end{array}$ & $\begin{array}{l}53 \\
86\end{array}$ \\
\hline $\begin{array}{c}-12(\mathrm{AC}) \\
332763\end{array}$ & $\begin{array}{l}\text { I. Iquor } \\
\text { Santue }\end{array}$ & $\begin{array}{r}94 \\
121\end{array}$ & $\begin{array}{r}87 \\
161\end{array}$ & $\begin{array}{r}79 \\
240\end{array}$ & $\begin{array}{r}89 \\
139\end{array}$ & $\begin{array}{r}66 \\
128\end{array}$ & $\begin{array}{r}82 \\
114\end{array}$ & $\begin{array}{l}88 \\
85\end{array}$ & $\begin{array}{r}86 \\
126\end{array}$ & - \\
\hline $\begin{array}{l}13(\mathrm{OT}) \\
333469\end{array}$ & $\begin{array}{l}\text { L. Squor } \\
\text { Sanguo }\end{array}$ & $\begin{array}{r}93 \\
126\end{array}$ & $\begin{array}{r}93 \\
163\end{array}$ & $\begin{array}{r}93 \\
148\end{array}$ & $\begin{array}{r}88 \\
133\end{array}$ & $\begin{array}{r}91 \\
128\end{array}$ & $\begin{array}{r}9 ! \\
206\end{array}$ & $\begin{array}{r}93 \\
122\end{array}$ & $\begin{array}{r}93 \\
211\end{array}$ & - \\
\hline $\begin{array}{l}\text { NODIAS } \\
\text { ALTER. }\end{array}$ & $\begin{array}{l}\text { Ifquor } \\
\text { Sangue }\end{array}$ & $\begin{array}{r}72,0 \\
116,4\end{array}$ & $\begin{array}{r}70,4 \\
137,6\end{array}$ & $\begin{array}{c}67,0 \\
116,25\end{array}$ & $\begin{array}{r}70,4 \\
115,2\end{array}$ & $\begin{array}{r}66,4 \\
104,2\end{array}$ & $\begin{array}{l}69,4 \\
99,8\end{array}$ & $\begin{array}{l}69,4 \\
98,4\end{array}$ & $\begin{array}{r}66,2 \\
111,6\end{array}$ & - \\
\hline $\begin{array}{l}\text { MEDIAS } \\
\text { GERAIS }\end{array}$ & $\begin{array}{l}\text { Liquor } \\
\text { Sangue }\end{array}$ & $\begin{array}{r}65,0 \\
107,2\end{array}$ & $\begin{array}{r}68,1 \\
135,8\end{array}$ & $\begin{array}{r}61,1 \\
105,9\end{array}$ & $\begin{array}{r}64,1 \\
103,4\end{array}$ & $\begin{array}{r}61,5 \\
107,4\end{array}$ & $\begin{array}{l}60,5 \\
96,0\end{array}$ & $\begin{array}{l}91,8 \\
97,2\end{array}$ & $\begin{array}{r}56,9 \\
106,6\end{array}$ & $m$ \\
\hline
\end{tabular}

Quadro 2 - Resultado das dosagens de glicose no líquor e no sangue, nos pacientes do grupo 2 (nos quais foram injetadas $10 \mathrm{~g}$ de glicose). Os casos $4,5,6,7$ e 8 apresentavam líquor normal; os restantes tinham alterações liquóricas. 
tes de sangue e líquor na ordem cronológica habitual (quadro 1). Ao determinarmos o êrro do método de dosagem, já havíamos verificado que as oscilações médias dêste grupo poderiam decorrer ùnicamente das falhas do método. Todavia, para apurar melhor esta questão, procuramos verificar se havia regressão linear entre as taxas liquóricas médias e o tempo da colheita, até o fim da primeira hora.

Obtivemos a seguinte equação de regressão: $y=52,799-0,035 x$. Submetido o coeficiente de regressão à prova de nulidade (Snedecor 10b), foi comprovada sua não significância: $t=0,598$ (o valor de $t$ para 3 graus de liberdade, ao nível fiducial de $5 \%$, é 3,182 ).

Evolução da glicorraquia no grupo 2 - Nesta série de 10 casos foram injetadas $10 \mathrm{~g}$ de glicose na veia. Já havíamos verificado que as oscilações médias do grupo se situavam dentro das próprias ao êrro experimental. No entanto, tendo-se verificado (quadro 2) que as dosagens médias do subgrupo $\mathrm{A}$ e dos 10 casos em conjunto, revelavam pequena elevação 15 minutos após a injeção, procuramos verificar se essas duas médias diferiam significativamente. Outrossim, como no grupo 1, investigamos se havia relação entre as taxas de glicorraquia e o momento da colheita, durante a primeira hora (5 primeiros valores), seja no total de 10 casos, seja nos subgrupos A (com líquor normal) e B (com líquor alterado).

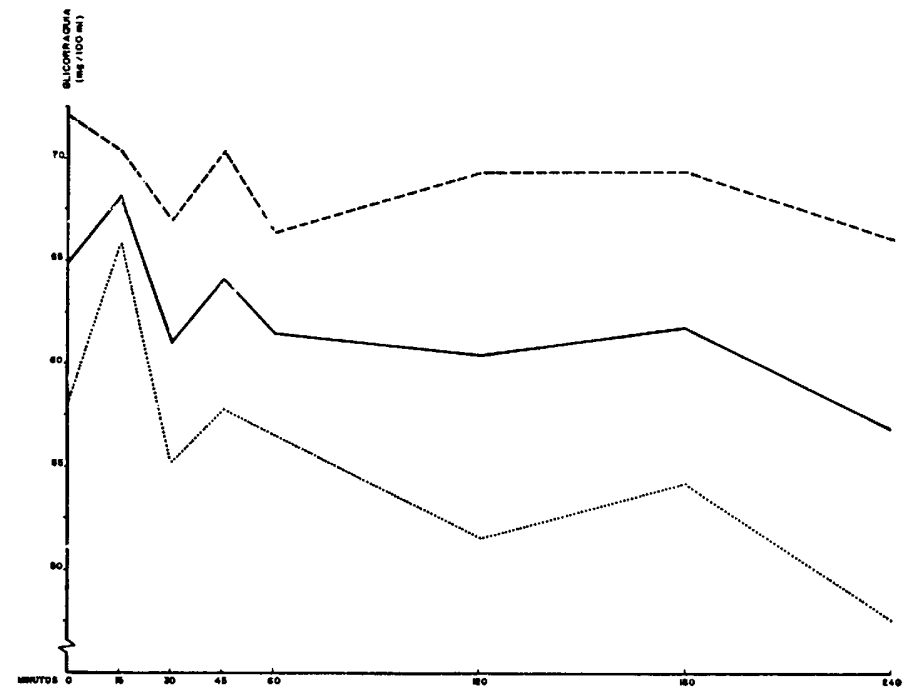

Fig. 1 - Evolução da glicorraquia no grupo 2 (após injeção intravenosa de $10 \mathrm{~g}$ de glicose): médias em 5 pacientes com líquor normal (em pontilhado), em 5 pacientes com líquor alterado (linha interrompida) e no conjunto de 10 pacientes (linha contínua). 
Aplicado o teste $t$ ì diferença entre a média inicial e a correspondente aos 15 minutos no conjunto de 10 casos, verificamos sua não significância $(t=0,313)$; o mesmo foi observado no subgrupo $A(t=0,475)$. Também não foi significante, do ponto de vista estatístico, a diferença entre as médias correspondentes aos 0 e 30 minutos no conjunto de 10 casos do grupo $2(t=0,530)$. Obtivemos a seguinte equação de regressão para o total de 10 casos: $y=66,150-0,073 x$; submetendo o coeficiente de regressão à prova de nulidade, comprovamos sua não significância $(t=1,331)$. Calculando as equações de regressão isoladamente para os subgrupos $\mathrm{A}$ e $\mathrm{B}$, obtivemos, respectivamente: $y \mathrm{~A}=60,840-0,072 x \mathrm{e}$ $y \mathrm{~B}=71,490-0,075 x$ (fig. 1). Ambos os coeficientes de regressão demonstraram-se nulos. Note-se, aliás, que todos os coeficientes de regressão foram negativos, indicando, pois, tendência ao decréscimo da glicorraquia após a administração intravenosa de $10 \mathrm{~g}$ de glicose, resultado êste francamente inesperado. Contudo, êste fato, desprovido de significado estatístico, pode resultar apenas do acaso.

Logo, a injeção intravenosa de $10 \mathrm{~g}$ de glicose não determinou alteração significativa da glicorraquia.

Evolução da glicorraquia no grupo 3 - Esta série, composta de 20 casos, foi subdividida em dois subgrupos, A (com líquor normal) e B (com líquor alterado). Já havíamos verificado que as oscilações médias

\begin{tabular}{|c|c|c|c|c|c|c|c|c|c|c|}
\hline \multirow{2}{*}{$\begin{array}{l}\text { CASO } \\
\text { RBSG.HC }\end{array}$} & \multirow[t]{2}{*}{ HUMORES } & \multicolumn{9}{|c|}{ AMCSTPAL (tempo om minutos) } \\
\hline & & 0 & 15 & 30 & $\overline{45}$ & 60 & 120 & 180 & 240 & $24 \mathrm{hs}$. \\
\hline $\begin{array}{l}14(0 S) \\
222431\end{array}$ & $\begin{array}{l}\text { Liquor } \\
\text { Sartue }\end{array}$ & $\begin{array}{r}57 \\
100\end{array}$ & $\begin{array}{r}66 \\
185\end{array}$ & $\begin{array}{r}63 \\
170\end{array}$ & $\begin{array}{l}80 \\
77\end{array}$ & $\begin{array}{l}83 \\
70\end{array}$ & $\begin{array}{l}80 \\
8.3\end{array}$ & $\begin{array}{r}66 \\
106\end{array}$ & $\begin{array}{r}78 \\
106\end{array}$ & $=$ \\
\hline $\begin{array}{l}16(\mathrm{JV}) \\
207236\end{array}$ & $\begin{array}{l}\text { Lfquor } \\
\text { Sangue }\end{array}$ & $\begin{array}{r}59 \\
124\end{array}$ & $\begin{array}{r}68 \\
287\end{array}$ & $\begin{array}{r}78 \\
157\end{array}$ & $\begin{array}{l}76 \\
90\end{array}$ & $\begin{array}{l}83 \\
83\end{array}$ & $\begin{array}{r}110 \\
97\end{array}$ & $\begin{array}{l}71 \\
94\end{array}$ & $\begin{array}{r}57 \\
116\end{array}$ & 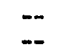 \\
\hline $\begin{array}{l}18 \text { (HG) } \\
220169\end{array}$ & $\begin{array}{l}\text { Liquor } \\
\text { Sangue }\end{array}$ & $\begin{array}{r}66 \\
103\end{array}$ & $\begin{array}{r}63 \\
269\end{array}$ & $\begin{array}{r}66 \\
212\end{array}$ & $\begin{array}{r}71 \\
148\end{array}$ & $\begin{array}{r}74 \\
129\end{array}$ & $\begin{array}{l}82 \\
75\end{array}$ & $\begin{array}{l}85 \\
77\end{array}$ & $\begin{array}{r}78 \\
100\end{array}$ & $\begin{array}{l}55 \\
88\end{array}$ \\
\hline $\begin{array}{l}17 \text { (JLR) } \\
150028\end{array}$ & $\begin{array}{l}\text { Liquor } \\
\text { Sangue }\end{array}$ & $\begin{array}{l}57 \\
94\end{array}$ & $\begin{array}{r}59 \\
307\end{array}$ & $\begin{array}{r}66 \\
278\end{array}$ & $\begin{array}{r}71 \\
231\end{array}$ & $\begin{array}{r}61 \\
201\end{array}$ & $\begin{array}{r}89 \\
106\end{array}$ & $\begin{array}{l}83 \\
75\end{array}$ & $\begin{array}{l}68 \\
91\end{array}$ & $\begin{array}{l}48 \\
83\end{array}$ \\
\hline $\begin{array}{l}18 \text { (HR) } \\
237778\end{array}$ & $\begin{array}{l}\text { Liquor } \\
\text { Sangue }\end{array}$ & $\begin{array}{r}63 \\
106\end{array}$ & $\begin{array}{r}66 \\
373\end{array}$ & $\begin{array}{r}63 \\
307\end{array}$ & $\begin{array}{r}71 \\
231\end{array}$ & $\begin{array}{r}75 \\
119\end{array}$ & $\begin{array}{l}71 \\
91\end{array}$ & $\begin{array}{l}63 \\
91\end{array}$ & $\begin{array}{l}66 \\
--\end{array}$ & $=$ \\
\hline $\begin{array}{l}19 \text { (ALS) } \\
227365\end{array}$ & $\begin{array}{l}\text { Lfquor } \\
\text { Sangue }\end{array}$ & $\begin{array}{l}54 \\
83\end{array}$ & $\begin{array}{r}52 \\
190\end{array}$ & $\begin{array}{r}52 \\
153\end{array}$ & $\begin{array}{r}56 \\
144\end{array}$ & $\begin{array}{r}56 \\
133\end{array}$ & $\begin{array}{l}65 \\
97\end{array}$ & $\begin{array}{l}65 \\
90\end{array}$ & $\begin{array}{l}61 \\
95\end{array}$ & $\begin{array}{l}42 \\
88\end{array}$ \\
\hline $\begin{array}{l}20(\mathrm{HB}) \\
141108\end{array}$ & $\begin{array}{l}\text { Liquor } \\
\text { Sanguo }\end{array}$ & $\begin{array}{l}46 \\
80\end{array}$ & $\begin{array}{r}48 \\
253\end{array}$ & $\begin{array}{r}48 \\
231\end{array}$ & $\begin{array}{r}40 \\
170\end{array}$ & $\begin{array}{r}53 \\
195\end{array}$ & $\begin{array}{l}57 \\
85\end{array}$ & $\begin{array}{l}63 \\
94\end{array}$ & $\begin{array}{l}63 \\
65\end{array}$ & $\begin{array}{l}54 \\
84\end{array}$ \\
\hline $\begin{array}{l}21 \quad(G M) \\
244061\end{array}$ & $\begin{array}{l}\text { LIquor } \\
\text { Senguse }\end{array}$ & $\begin{array}{r}71 \\
109\end{array}$ & $\begin{array}{r}76 \\
261\end{array}$ & $\begin{array}{r}71 \\
161\end{array}$ & $\begin{array}{r}83 \\
116\end{array}$ & $\begin{array}{r}81 \\
122\end{array}$ & $\begin{array}{l}65 \\
91\end{array}$ & $\begin{array}{r}67 \\
101\end{array}$ & $\begin{array}{r}66 \\
103\end{array}$ & $\begin{array}{r}59 \\
104\end{array}$ \\
\hline $\begin{array}{l}22 \text { (IB) } \\
224623\end{array}$ & $\begin{array}{l}\text { Llquor } \\
\text { Sanque }\end{array}$ & $\begin{array}{r}54 \\
175\end{array}$ & $\begin{array}{r}54 \\
350\end{array}$ & $\begin{array}{r}63 \\
287\end{array}$ & $\begin{array}{r}66 \\
269\end{array}$ & $\begin{array}{r}63 \\
278\end{array}$ & $\begin{array}{r}68 \\
100\end{array}$ & $\begin{array}{l}73 \\
88\end{array}$ & $\begin{array}{l}76 \\
91\end{array}$ & $=$ \\
\hline $\begin{array}{l}23 \text { (GB) } \\
256076\end{array}$ & $\begin{array}{l}\text { Liquor } \\
\text { Sangue }\end{array}$ & $\begin{array}{r}68 \\
104\end{array}$ & $\begin{array}{r}76 \\
292\end{array}$ & $\begin{array}{r}83 \\
224\end{array}$ & $\begin{array}{r}89 \\
239\end{array}$ & $\begin{array}{r}95 \\
104\end{array}$ & $\begin{array}{l}91 \\
66\end{array}$ & $\begin{array}{l}86 \\
72\end{array}$ & $\begin{array}{l}76 \\
97\end{array}$ & -- \\
\hline MEUIAS & $\begin{array}{l}\text { Liquor } \\
\text { Sangue }\end{array}$ & $\begin{array}{r}59,5 \\
106,8\end{array}$ & $\begin{array}{r}62,8 \\
276,7\end{array}$ & $\begin{array}{r}65,3 \\
218,0\end{array}$ & $\begin{array}{r}70,3 \\
159,5\end{array}$ & $\begin{array}{r}72,4 \\
143,4\end{array}$ & $\begin{array}{l}77,8 \\
87,9\end{array}$ & $\begin{array}{l}72,2 \\
88,8\end{array}$ & $\begin{array}{l}68,9 \\
96,0\end{array}$ & -- \\
\hline
\end{tabular}

Quadro 3 - Resultado das dosagens de glicose no líquor e no sangue, nos pacientes do grupo 3, com líquor normal (nos quais foram injetadas $50 \mathrm{~g}$ de glicose na veia). 
do grupo diferiam significantemente das atribuíveis exclusivamente ao érro experimental. Para uniformidade de pesquisa, submetemos o material (quadros 3 e 4) às mesmas provas estatísticas aplicadas ao grupo 2 . Assim, investigamos a significância das diferenças entre os valores médios iniciais e os correspondentes às colheitas realizadas 15 e 30 minutos após a injeçã̃o de $50 \mathrm{~g}$ de glicose. Finalmente, estabelecemos a equação de

\begin{tabular}{|c|c|c|c|c|c|c|c|c|c|c|}
\hline \multirow{2}{*}{$\begin{array}{l}\text { OASC } \\
\text { RES.HC }\end{array}$} & \multirow[t]{2}{*}{ НUHORES } & \multicolumn{9}{|c|}{ AHOSTRAS (tempo em minutos) } \\
\hline & & 0 & 15 & 30 & $\overline{45}$ & 60 & 120 & 130 & 240 & $24 \mathrm{hs}$. \\
\hline $\begin{array}{l}24(E L) \\
21917 S\end{array}$ & $\begin{array}{l}\text { Liquor } \\
\text { Sangue }\end{array}$ & $\begin{array}{l}35 \\
67\end{array}$ & $\begin{array}{r}52 \\
238\end{array}$ & $\begin{array}{r}72 \\
206\end{array}$ & $\begin{array}{r}86 \\
163\end{array}$ & $\begin{array}{r}87 \\
129\end{array}$ & $\begin{array}{l}84 \\
70\end{array}$ & $\begin{array}{l}70 \\
66\end{array}$ & $\begin{array}{l}70 \\
97\end{array}$ & $\begin{array}{l}-- \\
--\end{array}$ \\
\hline 25 (LRR) & Líquor & 48 & 66 & 84 & 110 & 118 & 99 & 74 & 68 & - \\
\hline 221942 & jangue & 91 & 343 & 225 & 225 & 148 & 65 & 60 & 88 & -- \\
\hline 26 (JS) & Liquor & 75 & 77 & 77 & 77 & 86 & 86 & 81 & 79 & 66 \\
\hline 225512 & Sangue & 129 & 208 & 156 & 129 & 137 & 119 & 97 & 91 & 37 \\
\hline 27 (LAS) & Llquor & 66 & 96 & 94 & 81 & 77 & 75 & 70 & $B 9$ & 58 \\
\hline 224125 & Sanguo & 106 & 157 & 163 & 128 & 309 & 79 & 88 & 77 & 216 \\
\hline $28(A L C)$ & Llquor & 68 & 66 & 75 & 77 & 77 & 79 & 77 & 70 & 58 \\
\hline 207520 & Sangue & 80 & 225 & 170 & 103 & 51 & 51 & 77 & 83 & 72 \\
\hline 29 (LC) & Liquor & 72 & 72 & 79 & 79 & 84 & 81 & 75 & 70 & 63 \\
\hline $1804158^{\circ}$ & Sangue & 85 & 319 & 201 & 119 & 116 & 85 & 94 & 100 & 89 \\
\hline $3 \cap($ 뱅) & Lfquor & 46 & 44 & 50 & 50 & 48 & 54 & 57 & 59 & 54 \\
\hline 179028 & Sangue & 66 & 180 & 133 & 91 & 103 & 62 & 53 & 75 & 88 \\
\hline 31 (RHS) & Llquor & 56 & 57 & 59 & 59 & 66 & 54 & 18 & 44 & - \\
\hline 227034 & Sangue & 76 & 261 & 201 & 148 & 100 & 72 & 83 & 97 & $-\infty$ \\
\hline 32 (ON) & Llquor & 57 & 58 & 61 & 61 & 63 & 73 & 83 & 76 & - \\
\hline $205(101$ & ŝangere & 94 & 261 & 331 & 225 & 144 & 80 & 100 & 80 & -- \\
\hline $33(\Lambda R)$ & LEquor & 54 & 68 & 30 & 79 & 94 & 91 & $6 B$ & 68 & - \\
\hline 201384 & Saciguo & 88 & 231 & 157 & 119 & 122 & 83 & 85 & 77 & -- \\
\hline VEDIAS & $\begin{array}{l}\text { Liquor } \\
\text { Sangue }\end{array}$ & $\begin{array}{l}57,7 \\
88,0\end{array}$ & $\begin{array}{r}65,1 \\
242,1\end{array}$ & $\begin{array}{r}72,1 \\
134,3\end{array}$ & $\begin{array}{r}75,9 \\
175,1\end{array}$ & $\begin{array}{r}80,0 \\
119,9\end{array}$ & $\begin{array}{l}77,6 \\
76,6\end{array}$ & $\begin{array}{l}70,1 \\
80,2\end{array}$ & $\begin{array}{l}69,3 \\
86,5\end{array}$ & -- \\
\hline
\end{tabular}

Quadro 4 - Resultado das dosagens de glicose no líquor c no sangue, nos pacientes do grupo 3, com líquor alterado (nos quais foram injetadas $50 \mathrm{~g}$ de glicose na veia).

regressão linear da primeira hora, nos dois subgrupos, e comparamos os respectivos coeficientes; utilizamos nestes cálculos apenas os 5 primeiros valores médios, correspondentes à primeira hora, devido a têrmos obser. vado gràficamente (fig. 2) que, durante êsse período, os valores médios obtidos pareciam adaptar-se perfeitamente a uma linha reta.

Aplicando o teste $t$ às diferenças entre a média inicial e as correspondentes aos 15 e 30 minutos no conjunto de 20 casos, obtivemos os seguintes valores, respectivamente: 1,451 e 2,856. Êste último é significante ao nível de 5\%. Obtivemos as seguintes equações de regressão linear para os subgrupos A e B, respectivamente: $y \mathrm{~A}=59,400+0,222 x$ e $y \mathrm{~B}=59,090+0,369 x$. Submetidos à 
prova de nulidade, ambos os coeficientes de regressão mostraram-se altamente significantes $(t \mathrm{~A}=14,770$ e $t \mathrm{~B}=11,125)$. A seguir, aplicamos o teste $t$ à diferença entre os dois coeficientes de regressão, utilizando, para tanto, a fórmiula apresentada por Rider 11, e obtivemos o seguinte resultado, altamente significante: $t \mathrm{bA}-\mathrm{bB}=4,031$ (o valor para 6 graus de liberdade, ao nível fiducial de $5 \%$, é 2,447).

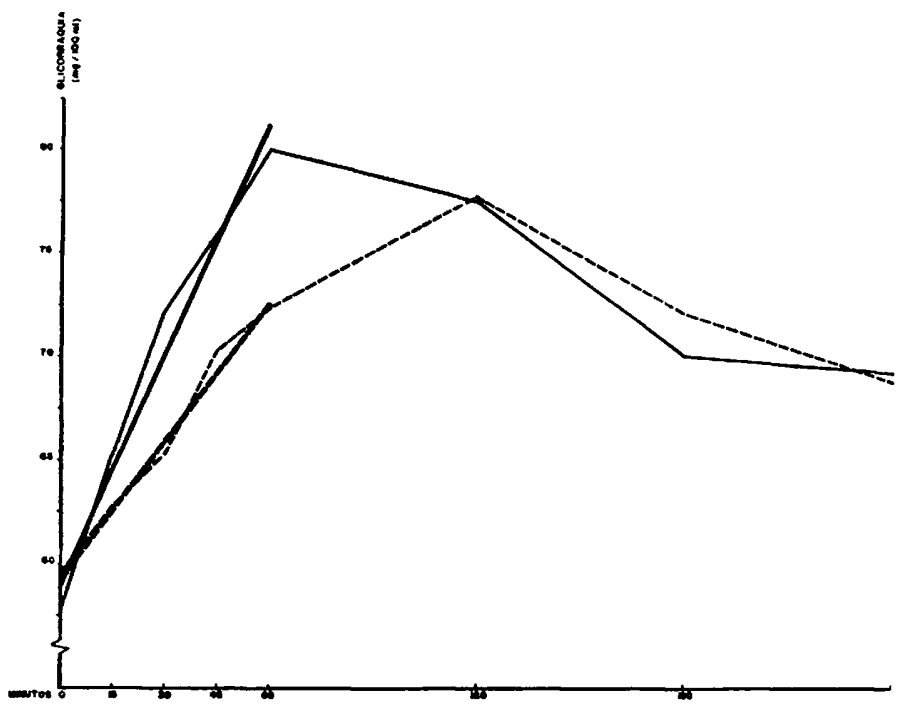

Fig. 2 - Evolução da glicorraquia no grupo 3 (após injeção intravenosa de $50 \mathrm{~g}$ de glicose): médias de 10 pacientes com líquor normal (linha interrompida) e de 10 pacientes com líquor alterado (linha contínua). Em linhas mais espêssas, a representação das respectivas equações lineares de regressão.

Logo, após a administração intravenosa de $50 \mathrm{~g}$ de glicose, obtém-se, durante a primeira hora, uma elevação regular das taxas da glicorraquia, a qual é mais intensa nos pacientes com líquor alterado. Por outras palavras, o teor de glicose se eleva mais ràpidamente e com maior amplitude nos pacientes com líquor alterado que nos casos com líquor normal. Tal conclusão era de esperar, visto que nos primeiros é clàssicamente admitida a ocorrência de rotura e, conseqüentemente, aumento da permeabilidade da barreira hemoliquórica.

Relação $L / S$ no grupo 2 - Calculamos os quocientes médios entre a glicorraquia e a glicemia (L/S) nos subgrupos A e B desta série. Obtivemos, para as amostras colhidas dos 15 aos 120 minutos, os seguintes valores: I) para o subgrupo $A, \quad 0,490,57 \quad 0,63 \quad 0,51 \quad 0,60$; II) para o subgrupo B, $0,51 \quad 0,58 \quad 0,61 \quad 0,64 \quad 0,70$. Nota-se que obedecem a 
uma ordem crescente e por isso calculamos as respectivas equações de regressão.

Obtivemos as seguintes equações de regressão linear para os subgrupos $A$ e $\mathrm{B}: \quad y \mathrm{~A}=0,52544+0,00064 x$ e $y \mathrm{~B}=0,51404+0,00174 x$. Aplicando a prova de nulidade aos coeficientes de regressão, obtivemos os seguintes valores: $t \mathrm{~A}=$ $=0,843$ (não significante) e $t \mathrm{~B}=4,962$ (significante ao nível fiducial de $5 \%$ ). Sendo nulo um dos coeficientes, ficou prejudicado o estudo estatístico da difercnça entre ambos.

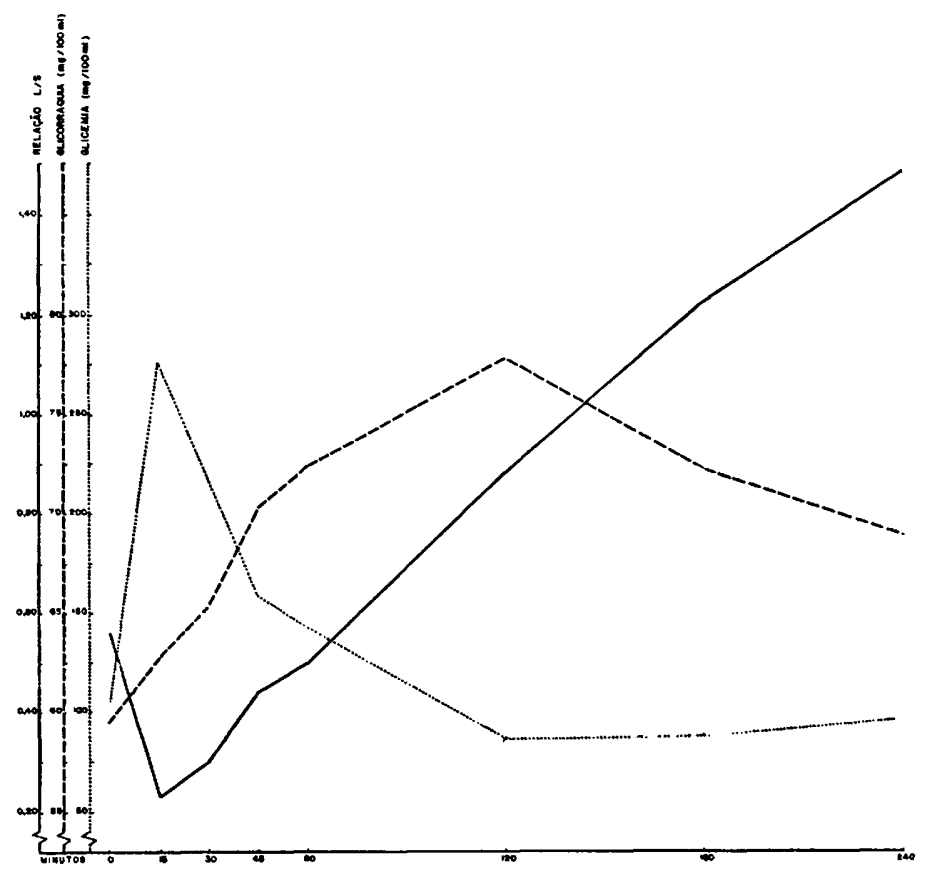

Fig. 3 - Evolução dos quocientes entre as taxas de líquor e sangue (linha contínua) após a injeção intravenosa de $50 \mathrm{~g}$ de glicose, em 10 pacientes com líquor normal (subgrupo $3 \mathrm{~A}$ ).

Por conseguinte, os quocientes L/S elevaram-se progressiva e uniformemente, nos pacientes com líquor alterado, entre 15 e 120 minutos após a injeção de $10 \mathrm{~g}$ de glicose na veia; nos indivíduos com líquor normal as modificações observadas nesse quociente podem ser consideradas puramente casuais.

Relação $L / S$ no grupo 3 - Calculamos os quocientes médios entre a glicorraquia e a glicemia (L/S) nos subgrupos A e B desta série (figs. 3 e 4). Observa-se imediatamente que, a partir dos 15 minutos após a in. jeção intravenosa de $50 \mathrm{~g}$ de glicose, êsses quocientes se elevam até os 120 
minutos, pois, ao passo que a glicemia decresce, a glicorraquia aumenta. Nesse periodo, as curvas se aproximavam estreitamente da linha reta.

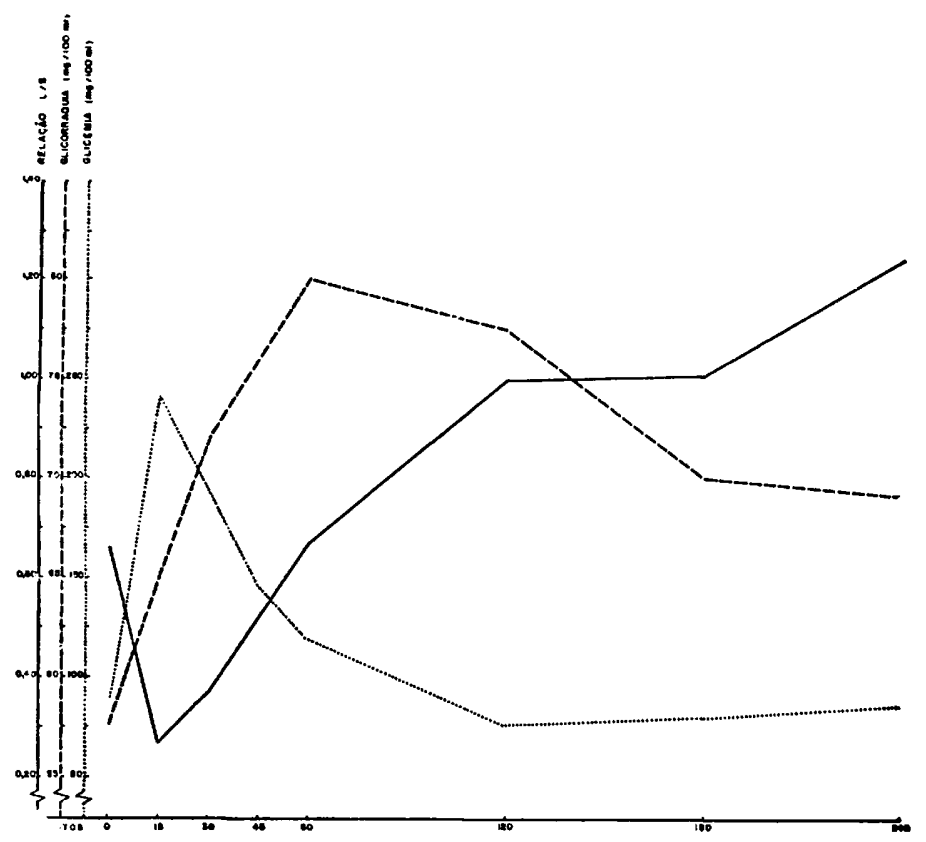

Fig. 4 - Evolução dos quocientes entre as taxas de líquor e sangue (linha contínua) após injeção intravenosa de $50 \mathrm{~g}$ de glicose, em 10 pacientes com líquor alterado (subgrupo $3 \mathrm{~B}$ ).

Obtivemos as seguintes equações de regressão linear para os subgrupos A e $\mathrm{B}: \quad y \mathrm{~A}=0,1308+0,0063 x$ e $y \mathrm{~B}=0,1846+0,0071 x$ (fig. 5). Aplicando a prova de nulidade aos coeficientes de regressão, obtivemos os seguintes valores: $t \mathrm{~A}=26,022$ e $t \mathrm{~B}=13,207$ (ambos altamente significantes). A aplicação do teste $t$ à diferença entre os dois coeficientes de regressão revelou, contudo, sua não significância $(t=1,356)$.

Logo, os quocientes L/S elevaram-se progressiva e uniformemente, tanto nos pacientes com líquor normal, como naqueles com líquor alterado, no período entre 15 e 120 minutos após a injeção intravenosa de $50 \mathrm{~g}$ de glicose. Embora, como era esperado, a elevação fôsse maior no subgrupo $B$, essa diferença careceu de significado estatístico.

Relação $L / S$ inicial - Calculamos os quocientes entre as taxas de glicose no líquor e no sangue, determinadas antes da injeção de glicose, nos pacientes dos grupos 2 e 3 (valores correspondentes a 0 minuto nos quadros 2,3 e 4 ; notar que, em um caso do subgrupo $2 \mathrm{~A}$, não dispomos da taxa inicial da glicemia). 


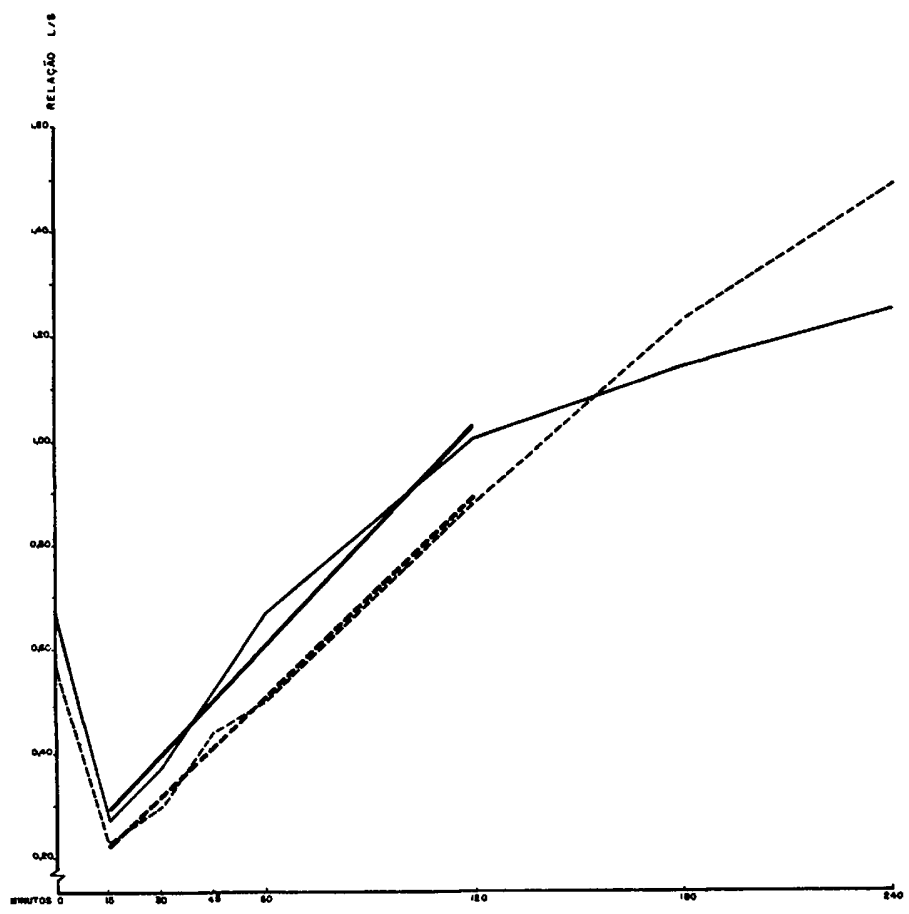

Fig. 5 - Comparação do comportamento dos quocientes entre as taxas de líquor e sangue, em 10 pacientes com líquor normal (linha interrompida) e 10 com líquor alterado (linha contínua), após injeção intravenosa de $50 \mathrm{~g}$ de glicose. Em linhas mais espêssas, a representação das respectivas equações lineares de regressão.

Em 14 casos com líquor normal obtivemos a seguinte média para a relação L/S inicial: $0,577 \pm 0,115$. Em 15 casos com líquor alterado o quociente médio foi de $0,654 \pm 0,135$. A aplicação do teste $t$ à diferença entre estas duas médias revelou sua não significância $(t=1,644)$. Por conseguinte, calculamos o quociente $\mathrm{L} / \mathrm{S}$ médio no total de 29 casos, obtendo o valor $0,617 \pm 0,129$. Baseados neste resultado, calculamos o intervalo fiducial de $95 \%$, de Fisher (Snedecor $\left.{ }^{10}, 2.12\right)$, obtendo o seguinte valor para a média da população: $m=0,617 \pm$ $\pm 0,049$, cujos limites fiduciais, para $t=2,045$, serão 0,568 e 0,666 . Por outro lado, conhecido o valor da média da amostra, podemos dizer que há apenas a probabilidade 3:1000 de que se encontrem valores do quociente $\mathrm{L} / \mathrm{S}$ inicial fora dos limites 1,004 e 0,230 (o índice 1,13 encontrado por Polonovski e Duhot 1 , portanto, é altamente improvável, devendo ser considerado sob reserva).

$O$ quociente entre as taxas iniciais de glicose no líquor e no sangue não diferiu nos pacientes com líquor normal e alterado; seu valor médio, em 29 casos, foi de $0,617 \pm 0,129$. 
Comparação entre os valores iniciais da glicorraquia $e$ os subseqüentes à primeira hora no grupo 3 - $\mathrm{O}$ exame da figura 2 revela que, mesmo em prazo superior a 60 minutos após a injeção de $50 \mathrm{~g}$ de glicose, são obtidos valores da glicorraquia muito maiores que os iniciais. Aplicamos o teste $t$ no sentido de verificar se as médias correspondentes a 120 , 180 e 240 minutos, no conjunto de 20 casos do grupo 3 , diferiam da inicial. Além disso, em 10 casos dêsse grupo (quadros 3 e 4) comparamos a média dos valores iniciais da glicorraquia e da glicemia, com os obtidos 24 horas após a administração de glicose.

Obtivemos os seguintes valores para as diferenças entre a média inicial nos 20 casos do grupo 3, e as médias relativas aos 120, 180 e 240 minutos, respectivamente: $t 120-0=4,752 ; t 180-0=3,879 ; t 240-0=3,282$ (todos significantes ao nível fiducial de 5\%). Obtivemos, para os valores iniciais e de 24 horas da glicorraquia, as seguintes médias: $62,10 \pm 10,64$ e 56,200 $\pm 7,675$. A diferença entre essas médias não é significante $(\bar{t}=1,422)$. Para a glicemia, obtivemos as seguintes médias: inicial $93,40 . \pm 18,58$; de 24 horas $89,80 \pm 12,08$. Também não é significante a diferença entre estas médias $(t=0,514)$.

Portanto, a injeção de $50 \mathrm{~g}$ de glicose na veia determinou elevação da glicorraquia que perdurou pelo menos até 4 horas; entretanto, não houve diferença entre os valores da glicemia e da glicorraquia obtidos antes e 24 horas após a administração dessa quantidade de glicose.

\section{RESUMO}

Com a finalidade de verificar a influência da administração intravenosa de glicose sôbre a glicorraquia, os autores, após avaliação do êrro do método de dosagem, estudaram particularmente os efeitos da injeção de glicose em três grupos de pacientes: grupo l, constituído por 3 casos que serviram de contrôle dos resultados; grupo 2, compreendendo 5 casos com líquor normal e 5 com líquor alterado; grupo 3 , composto de 10 casos com líquor normal e $10 \mathrm{com}$ líquor alterado. Nos pacientes do grupo 1 não foi injetada glicose; nos do grupo 2 foram administradas $10 \mathrm{~g}$ de glicose; os do grupo 3 receberam $50 \mathrm{~g}$ de glicose. 0 método utilizado consistiu no seguinte: a) colheita simultânea de sangue e líquor, em jejum, para determinação dos valores iniciais; b) injeção intravenosa de glicose; c) $15,30,45,60,120,180$ e 240 minutos após a injeção, colheita de novas amostras de sangue e líquor em cada paciente. Os resultados foram submetidos à análise estatística.

Foi verificado, inicialmente, que as oscilações dos valores médios obtidos nos grupos 1 e 2 eram comparáveis às devidas ao êrro experimental. Não foram significantes as diferenças entre a média inicial e os valores médios obtidos 15 e 30 minutos após a injeção de $10 \mathrm{~g}$ de glicose; nos pacientes que receberam $50 \mathrm{~g}$ de glicose, apenas após 30 minutos foi obtido um valor médio que diferia significantemente do inicial. Foram 
calculadas as equações de regressão linear relacionando a taxa de glicose no líquor com o momento da colheita. Foram estudados os quocientes entre as taxas de glicose no líquor e no sangue $(\mathrm{L} / \mathrm{S})$. Foi estudada a duração do efeito da injeção de glicose no grupo 3 .

Os autores chegaram às seguintes conclusões:

1. A injeção intravenosa de $10 \mathrm{~g}$ de glicose não determina alteração significativa da glicorraquia.

2. Após a administração intravenosa de $50 \mathrm{~g}$ de glicose, obtém-se, durante a primeira hora, uma elevação uniforme das taxas de glicorraquia, a qual é mais acentuada nos pacientes com líquor alterado, devido, provàvelmente, à maior permeabilidade da barreira hemoliquórica.

3. Os quocientes L/S elevaram-se progressivamente, nos pacientes com líquor alterado, entre 15 e 120 minutos após a injeção de $10 \mathrm{~g}$ de glicose na veia.

4. Os quocientes $\mathrm{L} / \mathrm{S}$ elevaram-se progressivamente, tanto nos pacientes com líquor normal como naqueles com líquor alterado, no período decorrente entre 15 e 120 minutos após a injeção intravenosa de $50 \mathrm{~g}$ de glicose; não foi significante a diferença de comportamento dos subgrupos.

5. O quociente médio entre as taxas iniciais de glicose no líquor e no sangue, em 29 casos, foi de $0,617 \pm 0,129$.

6. A elevação da glicorraquia após a injeção de $50 \mathrm{~g}$ de glicose persistiu durante 4 horas, pelo menos.

7. Não houve diferença entre os valores da glicemia e da glicorraquia obtidos antes e 24 horas após a administração intravenosa de $50 \mathrm{~g}$ de glicose.

SUMMARY

Study of the relations between blood and cerebrospinal fluid sugar contents.

In order to know the influence of intravenous injection of 50 per cent glucose solutions on the sugar contents of the cerebrospinal fluid. after estimating the error of the laboratory method the authors studied in detail such effects in three groups of patients: group 1, for controlling the results ( 3 cases); group 2, including 5 patients with normal and 5 with abnormal (presenting meningitic and/or parenchymatous changes) fluids; group 3 , including 10 patients with normal and 10 with abnormal fluids. 
In group 1 no glucose was injected; in group 2, 10 gm were administered; in group 3, $50 \mathrm{gm}$ were injected. The method of study consisted essentially of: a) simultaneous withdrawal of blood and fluid, in fasting conditions, for determination of the initial levels; b) intravenous injection of 50 percent glucose solution; c) $15,30,45,60,120,180$ and 240 minutes later, other samples of blood and fluid were withdrawn from each patient. The results were submitted to statistical analysis.

The oscilations of the average levels obtained in groups 1 and 2 were comparable to those due to the experimental error. The differences between the initial mean and the average contents 15 and 30 minutes after the injection of $10 \mathrm{gm}$ glucose were not statistically significant; in the patients receiving $50 \mathrm{gm}$, only after 30 minutes the average content differed significantly from the initial mean. The linear regression equations relating time to the blood and cerebrospinal fluid sugar contents were calculated. The ratios between fluid and blood sugar contents were analysed. In 10 cases of group 3 the blood and fluid sugar contents before and 24 hours after the glucose injection were compared.

The authors draw the following conclusions:

1. The intravenous injection of $10 \mathrm{gm}$ glucose does not influence significantly the cerebrospinal fluid sugar content.

2. After the intravenous injection of $50 \mathrm{gm}$ glucose it is observed, during the first hour, a uniform increase of the fluid sugar contents, mostly in the patients with abnormal fluid, probably owing to greater permeability of the blood-brain barrier.

3. The ratios between cerebrospinal fluid and blood sugar contents (CSF/B) showed progressive increase in the patients with abnormal fluid 15 to 120 minutes after the injection of $10 \mathrm{gm}$ glucose.

4. The CSF/B ratios showed progressive increase both in the patients with normal and abnormal fluid, 15 to 120 minutes after the injection of $50 \mathrm{gm}$ glucose; the difference between the regression coefficientes of these two groups was not significant.

5. The average initial ratio between the cerebrospinal fluid and the blood sugar contents, in 29 patients, was $0.617 \pm 0.129$.

6. The increase of the cerebrospinal fluid sugar content after the intravenous injection of $50 \mathrm{gm}$ glucose lasted 4 hours at least.

7. There was no significant difference between the blood and cerebrospinal fluid sugar contents before and 24 hours after the intravenous injection of $50 \mathrm{gm}$ glucose. 


\section{BIBLIOGRAFIA}

1. Polonovski, M. e Duhot, E. - Glycémie et glycorrachie. Presse Méd., 31: 60-62 (20 janeiro) 1923. 2. Wilcox, H. B., Lyttle, J. D. e Hearn, J. E. - The chemical composition of the spinal fluid. Am. J. Dis. Child,, 30:513-540 (outubro) 1925. 3. Cit. por Tcherkassov e Jolkver 4. 4. Tcherkassov, A. V. e Jolkver, E. E. - Le sucre du sang et du liquide céphalo-rachidien. J. Franç. de Pédiat., 11:350-364, 1935. 5. Becker, S. W. - The sugar and chloride content of cerebrospinal fluid with special reference to neurosyphilis. J. Lab. a. Clin. Med., 12: 43-52 (outubro) 1926-1927. 6. Dietel, F. G. - Untersuchungen über das Verhaltnis vom Blut- zum Liquor-zucker und über den diagnostischen Wert der Liquorzuckerbestimmung. Ztschr. f. d. ges. Neurol. u. Psychiat., 95:563-587, 1925. Cit. por Katzenelbogen, S.: The Cerebrospinal Fluid and its Relation to the Blood. Johns Hopkins Press, Baltimore, 1935, pág. 151. 7. Schjött, A. - The sugar curve in the cerebrospinal fluid after oral administration of glucose. Acta Psychiat. et Neurol., 6:247, 1931. Cit. por Katzenelbogen, S. (loc. cit.6). 8. Merritt, H. H. e Fremont-Smith, F. - The Cerebrospinal Fluid. Saunders, Filadelfia, 1947, pág. 43. 9. Folin, O. e Wu, H. - System of blood analysis; simplified and improved method for determination of sugar. J. Biol. Chem., 41:367 (março) 1920. 10. Snedecor, G. W. - a) Métodos de Estadística. Su aplicación a experimentos en Agricultura y Biologia. Trad. da $4^{a}$ edição americana. Acme Agency, Buenos Aires, 1948, capít. 10, secção 13; b) Idem, capít. 6, secção 9.11. Rider, P. R. - An Introduction to Modern Statistical Methods. John Wiley and Sons, Nova York, 1939, pág. 94.

$$
\text { Clínica Neurológica - Hospital das Clinicas - São Paulo. }
$$

